\title{
DINÂMICA DE NITROGÊNIO EM LODO DE ESGOTO SOB CONDIÇÕES DE ESTOCAGEM(1)
}

\author{
Charles Carneiro $^{(2)}$, Ana Paola Sottomaior ${ }^{(3)} \&$ Cleverson \\ Vitório Andreoli ${ }^{(4)}$
}

\begin{abstract}
RESUMO

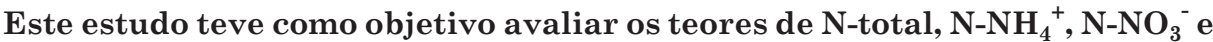
pH em colunas de lodo de esgoto anaeróbio alcalinizado e na solução percolada, simulando condições reais de armazenamento. $\mathrm{O}$ experimento foi desenvolvido em campo, no município de Curitiba (PR), em clima Cfb e pluviosidade de $197 \mathrm{~mm}$ durante o período de incubação, que correspondeu a 48 dias. Os fatores testados foram: três tipos de lodo (lodo alcalinizado coberto - LCC; lodo alcalinizado descoberto - LCD, e lodo bruto descoberto - LBD), quatro intervalos de incubação $(0 ; 15 ; 27$; e 48 dias) e cinco profundidades na coluna $(0-5 ; 5-20 ; 20-50 ; 50-80$; e composta 0-80 cm), com quatro repetições. O líquido percolado foi avaliado no $1^{\circ}, 5^{\circ}, 9^{\circ}, 13^{\circ}, 27^{\circ}, 36^{\circ}$ e $48^{\circ}$ dia. A alcalinização $(\mathrm{CaO})$ foi realizada à proporção de 50 \% da matéria seca (MS) do lodo. A cobertura (vedação com lona plástica e tampo de amianto em LCC) teve a finalidade de conter a volatilização de gases, especialmente amônia $\left(\mathrm{NH}_{3}\right)$. As condições do meio ocasionadas pela alcalinização do lodo reduziram significativamente a intensidade de mineralização de $\mathrm{N}$ e a nitrificação. Os lodos brutos e alcalinizados em condição de estocagem apresentaram perdas consideráveis de $\mathrm{N}^{-\mathrm{NH}_{4}}{ }_{4}$ e N-org. Os efeitos diluição, volatilização de $\mathrm{NH}_{3}$ e lixiviação organomineral conseqüentes da alcalinização ( $50 \%$ MS lodo) reduziram os teores de $\mathrm{N}$-total em até $50 \%$. O processo de alcalinização potencializou perdas de $\mathrm{N}$ por meio da volatilização de $\mathrm{NH}_{3}$ e lixiviação de $\mathrm{N}-\mathrm{NH}_{4}{ }^{+}$. Entretanto, a cobertura reduziu as perdas de $\mathrm{N}$ sob a forma de $\mathrm{NH}_{3}$.
\end{abstract}

Termos de indexação: amônio, nitrato, alcalinização, volatilização.

(1) Recebido para publicação em maio de 2004 e aprovado em agosto de 2005.

(2) Doutorando em Geologia Ambiental. Professor no IESC/PR. Rua: Eng. Rebouças 1376, Bairro Rebouças, CEP 80215-900 Curitiba (PR). Pesquisador da SANEPAR. E-mail: charlesc@sanepar.com.br

(3) Mestranda no Departamento de Solos e Engenharia Agrícola no Setor de Ciências Agrárias - UFPR. Rua dos Funcionários 1540 , Bairro Juvevê, CEP 80035-050 Curitiba (PR). E-mail: apsottomaior@hotmail.com

(4) Professor da Universidade Federal do Paraná - UFPR. Rua: Eng. Rebouças 1376, Bairro Rebouças, CEP 80215-900 Curitiba (PR). Pesquisador da SANEPAR. E-mail: c.andreoli@sanepar.com.br 


\title{
SUMMARY: NITROGENDYNAMICS IN SEWAGE SLUDGE STORED UNDER DISTINCT CONDITIONS
}

\begin{abstract}
This study aimed to evaluate total $\mathrm{N}, \mathrm{N}-\mathrm{NH}_{4}{ }^{+}$and $\mathrm{N}-\mathrm{NO}_{3}{ }^{-}$concentrations and $\mathrm{pH}$ in columns of alkalinized anaerobic sewage sludge and in percolated liquid, in simulation of real storage conditions. The experiment was conducted in Curitiba - Paraná state, in Cfb climate and rainfall of $197 \mathrm{~mm}$ during the incubation period (48 days). The tested factors were: 3 sludge types (covered alkalinized sludge - LCC; uncovered alkalinized sludge - LCD; and uncovered raw sludge - LBD), 4 incubation periods (0;15;27; and 48 days) and 5 column sampling depths (0-5; 5-20; 20-50; 50-80; and composite sample 0-80 cm), with 4 replications. The percolated liquid was evaluated on day 1, 5, 9, 13, 27, 36, and 48 . Alkalization ( $\mathrm{CaO}$ ) used was equivalent to $50 \%$ sludge dry matter. The cover (sealing with plastic blanket and asbestos cover in LCC) had the purpose to contain gas volatilization, especially ammonia $\left(\mathrm{NH}_{3}\right)$. The conditions of the environment caused by sludge alkalization significantly reduced the intensity of $N$ mineralization and nitrification. The raw and alkalinized stored sludges presented considerable $\mathrm{N}-\mathrm{NH}_{4}^{+}$and $\mathrm{N}$-org losses. The effect of dilution, $\mathrm{NH}_{3}$ volatilization and organic mineral leaching as result of the alkalization reduced $\mathrm{N}$-total concentration to $50 \%$. The alkalization process increased $\mathrm{N}$ losses through $\mathrm{NH}_{3}$ gas volatilization and $\mathrm{N}-\mathrm{NH}_{4}{ }^{+}$leaching, while the cover reduced $\mathrm{N}$ losses in the $\mathrm{NH}_{3}$ form.
\end{abstract}

Index terms: ammonium, nitrate, alcalinization, ammonia.

\section{INTRODUÇÃO}

A disposição agrícola de lodos de esgoto alcalinizados vem-se caracterizando como uma alternativa cada vez mais utilizada pelas companhias de saneamento, sobretudo por resolver, em parte, o problema da disposição final do resíduo, substituição à calagem e pela carga de nutrientes e matéria orgânica que concentra, podendo substituir parcialmente adubos agrícolas, principalmente nitrogenados.

Nos lodos domésticos o $\mathrm{N}$ tem uma importância diferenciada em razão dos elevados teores observados, assumindo significativa importância agronômica (Sommers \& Giordano, 1984; Ilhenfeld et al., 1999; Melo \& Marques, 2000; Andreoli et al., 2001). No entanto, devido ao seu grau de contaminação e potencial para disseminação de doenças, para viabilizar seu uso, é necessário que passe por um processo de higienização. A alcalinização é um processo de higienização amplamente utilizado, que consiste na adição de cal virgem $(\mathrm{CaO})$ ao lodo em proporções que variam de 30 a $50 \%$, dependendo do peso do lodo seco (Ilhenfeld et al., 1999b). O contato da cal com a água do lodo resulta em uma reação exotérmica, que promove a elevação da temperatura e $\mathrm{pH}$ e aumento da concentração de $\mathrm{NH}_{3}$ na mistura. Entretanto, comumente, antes da utilização, os lodos alcalinizados permanecem estocados nos pátios das estações de tratamento (ETEs) por períodos prolongados, sob influência de variáveis climáticas, podendo sofrer perdas de $\mathrm{N}$ bastante significativas.
Sob o aspecto financeiro as perdas de $\mathrm{N}$ podem significar um custo maior com fertilizantes nitrogenados; sob a ótica ambiental, problemas de contaminação dos cursos d'água. As formas nítricas são as mais facilmente lixiviáveis neste caso; acumulam-se nos lençóis mais próximos, alterando padrões normais de concentração e podendo comprometer a saúde humana. Estudos já realizados mostraram que a lixiviação de $\mathrm{NO}_{3}{ }^{-}$é significativamente influenciada pela fonte de $\mathrm{N}$ utilizada, pela capacidade de armazenamento de água no meio e pelo volume de água percolado (Pratt, 1984; Williams \& Kissel, 1991). Já as formas amoniacais, freqüentemente utilizadas como indicativo de poluição em ecossistemas aquáticos, estão diretamente relacionadas com a temperatura do meio, $\mathrm{pH}$ e capacidade de retenção de cátions (Hauck, 1984; Adams \& Martin, 1984). O N dos lodos pode ser perdido ainda por meio dos processos de desnitrificação, volatilização, fixação e, ou, erosão.

Este trabalho teve como objetivo avaliar as concentrações das formas nitrogenadas: $\mathrm{N}$-total, $\mathrm{N}$ $\mathrm{NH}_{4}{ }^{+}, \mathrm{N}-\mathrm{NO}_{3}{ }^{-}$e $\mathrm{pH}$ em diferentes profundidades de colunas de lodo de esgoto, quando em condição de estocagem e higienizado por meio de caleação.

\section{MATERIAIS E MÉTODOS}

O experimento foi desenvolvido no município de Curitiba (PR), clima Cfb (Köppen), em condições de 
campo. A temperatura média no período experimental foi de $15,3{ }^{\circ} \mathrm{C}$ e a precipitação pluvial foi de $197 \mathrm{~mm}$. O período de incubação foi de 48 dias, tempo médio de estocagem dos lodos nas ETEs de Curitiba que utilizam a alcalinização como processo de higienização.

As dimensões de cada unidade experimental foram: 0,63 $\mathrm{m}$ de diâmetro e $0,81 \mathrm{~m}$ de altura, para um volume de $0,25 \mathrm{~m}^{3}$ preenchido com lodo bruto ou lodo alcalinizado de acordo com o tratamento (extrato sólido). A coluna foi suspensa a $0,62 \mathrm{~m}$ de altura do solo para obtenção do extrato líquido percolado. As densidades para lodo bruto e para lodo alcalinizado foram de 0,90 e $0,98 \mathrm{~kg} \mathrm{~m}^{-3}$, respectivamente.

O lodo testado foi proveniente de reator anaeróbio de leito fluidizado (RALF - ETE - Cachoeira), disposto em leito de secagem durante 30 dias, para desaguamento. O lodo foi caracterizado analiticamente (Quadro 1).

O delineamento experimental utilizado foi o de blocos casualizados. O arranjo estatístico foi parcela subsubdividida para três fatores, sendo: fator A: três tipos de lodo (lodo alcalinizado coberto - LCC; lodo alcalinizado descoberto - LCD, e lodo bruto descoberto - LBD), fator B: quatros intervalos de tempo de incubação $(0 ; 15 ; 27$; e 48 dias) e fator $\mathrm{C}$ : cinco profundidades na coluna $(0-5 ; 5-20 ; 20-50$; 50-80; e composta 0-80 cm), com quatro repetições.

O processo de alcalinização envolveu a aplicação de óxido de $\mathrm{Ca}(\mathrm{CaO})$ na concentração de $50 \%$ em relação à matéria seca (MS) do lodo. A cobertura compreendeu vedação com lona plástica e tampo de amianto no tratamento LCC com a finalidade de conter a volatilização de gases, especialmente $\mathrm{NH}_{3}$, por meio da condensação do gás e retorno à massa de lodo.

O extrato percolado foi coletado de acordo com o regime pluviométrico, totalizando sete amostragens, a saber: $1^{\circ}, 5^{\circ}, 9^{\circ}, 13^{\circ}, 27^{\circ}, 36^{\circ}$ e $48^{\circ}$ dia.

As características analisadas nos extratos (sólido e líquido) foram: N-total e $\mathrm{N}-\mathrm{NH}_{4}{ }^{+}$(Kjeldahl titulação - adaptado de Tedesco et al., 1995), N-NO ${ }_{3}^{-}$ (extrato líquido: centrifugação e espectrofotometria de ultravioleta - Norman \& Stucki, 1981; Heinzmann et al., 1984; e extrato sólido - Norman \& Stucki, 1981; Heinzmann et al., 1984; Tedesco et al., 1995) e pH.
Os dados não apresentaram distribuição normal e o teste de Barttlet para verificação da homocedasticidade foi significativo. $O$ teste de Fischer $\left(\mathrm{F}_{0,05}\right)$ foi utilizado para analisar as variâncias. As médias foram comparadas pelo teste de Duncan $\left(\mathrm{D}_{0,05}\right)$. Foram consideradas significativas as correlações a $\geq 0,878$ (Pearson $\left._{0,05}\right)$; (Gomes, 1987; Triola, 1999).

\section{RESULTADOS E DISCUSSÃO}

\section{Teores de $\mathbf{N}$ total}

Em razão das composições diferenciadas dos tratamentos, obviamente, as médias dos teores de $\mathrm{N}$ total foram distintas. Em LCC e LCD, foram verificados teores que representaram, respectivamente, $49,5 \%\left(9,74 \mathrm{~g} \mathrm{~kg}^{-1}\right)$ e 54,1\% (10,7 $\left.\mathrm{g} \mathrm{kg}^{-1}\right)$ do N-total observado em LBD, 21,2 $\mathrm{g} \mathrm{kg}^{-1}$ (Figura 1a), muito embora estes valores estejam relativamente baixos, mas ainda comuns para lodos anaeróbios no Estado do Paraná. A diminuição da concentração é atribuída principalmente ao efeito diluição do processo de alcalinização (adição de $\mathrm{CaO} 50$ \% MS lodo), estimado em $33,3 \%$. As demais perdas de $\mathrm{N}$-total podem ter sido ocasionadas pela lixiviação organomineral, e, ou, pela volatilização de $\mathrm{NH}_{3}$ e compostos desnitrificados, por ocasião do aumento do $\mathrm{pH}$ decorrente da alcalinização, com valores próximos a 12 (Figura 1b). Os valores de $\mathrm{pH}$ normalmente observados em lodos brutos domésticos ficam entre 5 e 7 (Andreoli et al., 1999; Tchobanoglous et al., 2003), dependendo do material de origem.

Não foram observadas variações significativas nos teores de $\mathrm{N}$-total, considerando a profundidade e o tempo no perfil das colunas de lodo dentro dos tratamentos, indicando que as perdas mais expressivas aconteceram por ocasião do processo de alcalinização. As correlações testadas para os teores em meio sólido e lixívia não foram significativas.

O teor de N-total é uma das características comumente utilizadas para estimar a taxa de aplicação do biossólido ao solo. Neste sentido, obtémse o teor de $\mathrm{N}$ passível de mineralização e disponibilidade a determinada cultura durante seu ciclo, tendo, como caráter restritivo, a probabilidade de contaminação do lençol freático por $\mathrm{N}-\mathrm{NO}_{3}{ }^{-}$.

Quadro 1. Valores médios para a caracterização do lodo bruto

\begin{tabular}{|c|c|c|c|c|c|}
\hline Sólido total & C org. & $\mathrm{pH} \mathrm{H} \mathrm{H}_{2} \mathrm{O}$ & $\mathrm{N}-\mathrm{NO}_{3}$ & $\mathrm{~N}-\mathrm{NH}_{4}{ }^{+}$ & $\mathrm{N}$-total \\
\hline$\%$ & $\mathrm{~g} \mathrm{~kg}^{-1}$ & & + & $-\mathrm{mg} \mathrm{kg}^{-1}$ & +5 \\
\hline 32,0 & 200,11 & 4,92 & 0,354 & 114,69 & $19.594,00$ \\
\hline
\end{tabular}


O teor de N-total tem relação, direta e indiretamente, com a quantidade de $\mathrm{N}$ mineralizada, que, por sua vez, quando única fonte de $\mathrm{N}$ no sistema, determina o potencial de contaminação das águas e o suprimento de $\mathrm{N}$ satisfatório às plantas. Diante da ampla variabilidade nos valores de recuperação de $\mathrm{N}$, decorrente da implicação de inúmeras variáveis, a estimativa precisa desta determinação assume maior importância. Chae \& Tabatabai (1986), Munn et al. (2000); He et al. (2000); Zebarth et al. (2000), testando biossólidos, observaram valores bastante diferenciados para as taxas de recuperação do $\mathrm{N}$.

\section{Teores de $\mathrm{N}-\mathrm{NH}_{4}{ }^{+}$}

Os tratamentos LCC e LCD apresentaram baixos teores de $\mathrm{N}-\mathrm{NH}_{4}{ }^{+}$graças ao efeito diluição e adversidades do meio impostas pelo agente alcalinizante para a evolução do processo bioquímico de mineralização. Ademais, não se diferenciaram estatisticamente em relação à profundidade e ao período de tempo testado (Figura $2 \mathrm{a}$ e b).

O comportamento do lodos caleados com relação à amonificação foi semelhante. Embora nãosignificativa, houve uma redução temporal nos teores, evidenciando o efeito inibidor do $\mathrm{pH}$ elevado sobre a atividade de mineralização do N. Os valores verificados em LBD, relativamente superiores aos materiais alcalinizados, apresentaram aumento progressivo em razão do tempo e profundidade da coluna, com indicação também no extrato percolado (Quadro 2). A elevação temporal dos teores pode estar relacionada com o aumento do $\mathrm{pH}(\mathrm{r}=0,97)$ dentro de níveis tidos como ótimos e uma progressiva (a)

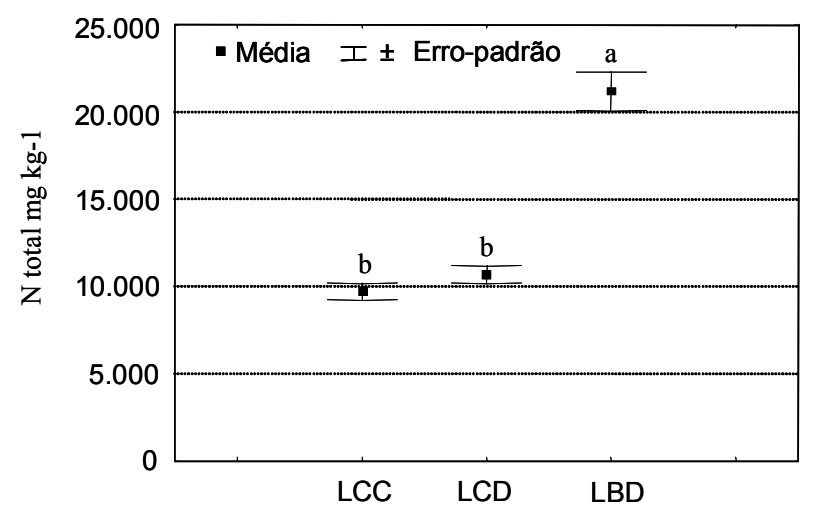

(b)

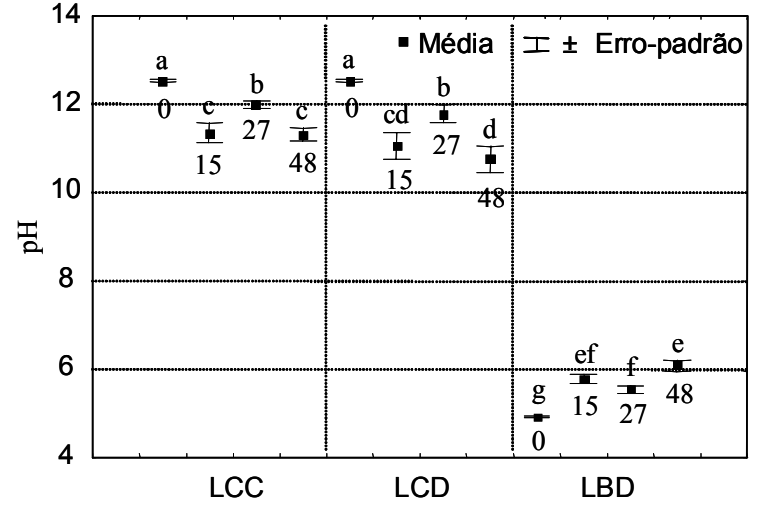

LCC - lodo alcalinizado coberto; LCD - lodo alcalinizado descoberto; LBD - lodo bruto descoberto.

Médias seguidas pela mesma letra não diferem estatisticamente pelo teste de Duncan ${ }_{(0,05)}$.

Figura 1. Teores médios de $\mathrm{N}$ total de acordo com o tipo de lodo (a) e valores de pH de acordo com o tipo de lodo e tempos de incubação de 0, 15, 27 e 48 dias (b).

(a)

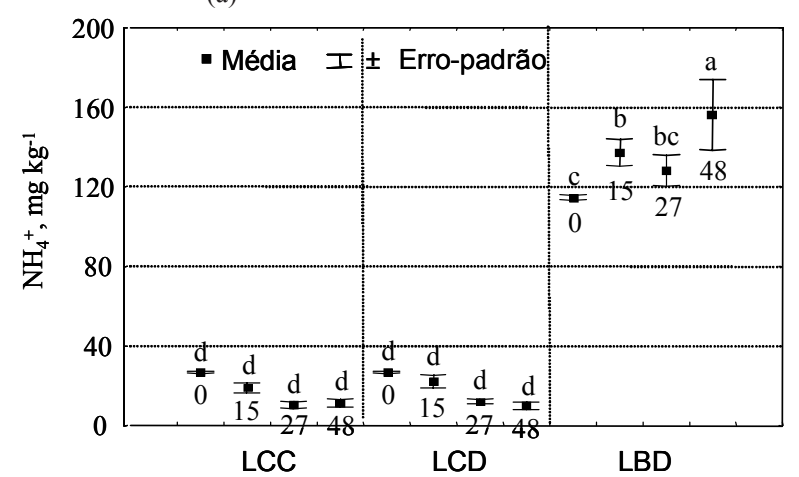

(b)

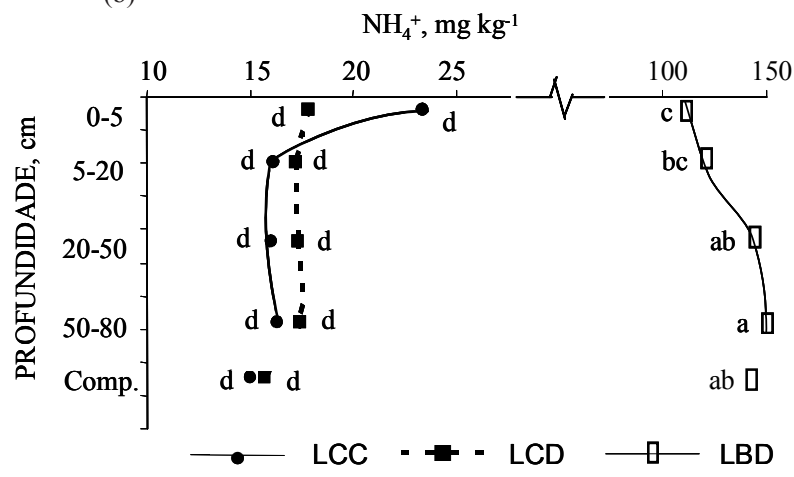

LCC - lodo alcalinizado coberto; LCD - lodo alcalinizado descoberto; LBD - lodo bruto descoberto. Comp: amostragem composta do perfil.

Médias seguidas pela mesma letra não diferem estatisticamente pelo teste de Duncan $(0,05)$.

Figura 2. Teores de $\mathrm{N}-\mathrm{NH}_{4}{ }^{+}$nos diferentes tipos de lodo de acordo com: (a) os tempos de incubação de 0, 15, 27 e 48 dias; (b) profundidade. 
adaptabilidade dos organismos decompositores. O aumento da temperatura dentro de níveis ótimos também eleva a taxa de hidrólise dos compostos (Brady, 1989).

Acredita-se que a produção de $\mathrm{NH}_{3}$ tenha sido potencializada pela alcalinização, embora não mensurada diretamente. Hauck (1984) menciona que a concentração de $\mathrm{NH}_{3}$ em solução aumenta exponencialmente para $\mathrm{pH}$ acima de 8 . A adição de $\mathrm{CaO}$ elevou o pH da mistura próximo a 12 , o que tornou o meio extremamente alcalino, condicionando a maior alteração de compostos amoniacais, como, por exemplo $\left(\mathrm{NH}_{4}\right)_{2} \mathrm{CO}_{3}$, aumentando consideravelmente a lixiviação de íons $\mathrm{N}-\mathrm{NH}_{4}{ }^{+}$e volatilização de $\mathrm{NH}_{3}$.

Observou-se, com a utilização da cobertura, uma retenção parcial dos gases amoniacais, por meio da condensação na parede interna do revestimento e conseqüente retorno à massa superficial do lodo, o que pode ser verificado pelos maiores teores de $\mathrm{N}-\mathrm{NH}_{4}{ }^{+}$, na camada de 0-5 cm, no tratamento LCC. Contudo, Andreoli \& Carneiro (2002), avaliando o comportamento de $\mathrm{N}-\mathrm{NH}_{4}{ }^{+}$em lodo, observaram uma eficiência maior na retenção de $\mathrm{NH}_{3}$ com a utilização de cobertura sobre leiras do resíduo.

\section{Teores de $\mathrm{N}-\mathrm{NO}_{3}{ }^{-}$}

Os teores de $\mathrm{N}-\mathrm{NO}_{3}{ }^{-}$em todos os tratamentos foram muito baixos $\left(<2,0 \mathrm{mg} \mathrm{kg}^{-1}\right)$ comparativamente a valores normalmente observados, muito embora LCC e LCD tenham apresentado teores superiores a LBD (Figura 3a). Ainda que pouco expressivas, foram observadas variações estatisticamente significativas nas concentrações de $\mathrm{N}_{-} \mathrm{NO}_{3}{ }^{-}$nos tratamentos LCC e LCD de acordo com o tempo e profundidade (Figura $3 \mathrm{a}$ e b).
Nos tratamentos alcalinizados, observou-se pequeno aumento inicial com a alcalinização, graças ao progressivo aumento de $\mathrm{pH}$; no entanto, com a elevação a valores acima de 11 , houve inibição acentuada do processo de nitrificação. O limite ácido teórico para a nitrificação é variável e próximo a 5,0, dependendo predominantemente do grau de tolerância das bactérias à acidez; o limite superior teria um ótimo até $\mathrm{pH}$ 8,0 aproximadamente, embora, não haja uma relação direta entre o pH e a taxa de nitrificação (Russel, 1973). Entretanto, em algumas situações específicas, é possível verificar nitrificações além destes limites.

Os teores para LCC mantiveram-se constantes nos tempos 15, 27 e 48 dias, pela não-ocorrência de lixiviação graças à presença de cobertura; e LCD, onde ocorreu lixiviação de $\mathrm{N}^{-\mathrm{NO}_{3}}{ }^{-}$(embora ínfima devida aos baixos teores na mistura), mostrou alguma diminuição temporal nestes teores.

É notório que o processo de nitrificação depende essencialmente de $\mathrm{pH}$ e oxigenação adequados para a efetividade das reações. Acredita-se que a desestabilização do meio percebida nos tratamentos LCC e LCD, com acréscimos no pH, salinidade, temperatura e concentração de $\mathrm{NH}_{3}$ devidos à alcalinização, foi determinante para a inibição da nitrificação, sendo notadamente $\mathrm{pH}$ o fator com maior influência. A desnitrificação também deve ser considerada como fator de redução dos teores de $\mathrm{N}-\mathrm{NO}_{3}{ }_{3}^{-}$, especialmente em se tratando de lodo digerido anaerobiamente e, por vezes, de tratamentos com umidade elevada, o que significa baixo $\mathrm{O}_{2}$. Em relação à profundidade, LCD confirmou maior lixiviação de $\mathrm{N}_{-} \mathrm{NO}_{3}{ }^{-}$em superfície resultante da nãocobertura; já LCC mostrou alguma variação vertical

Quadro 2. Concentrações de N-total, $\mathrm{N}_{-} \mathrm{NH}_{4}{ }^{+}$e N-NO ${ }_{3}{ }^{-}$nos volumes percolados no perfil do lodo de acordo com os tratamentos aplicados e período experimental

\begin{tabular}{|c|c|c|c|c|c|c|c|c|c|}
\hline \multirow{2}{*}{ TI(1) } & \multirow{2}{*}{$\begin{array}{c}\text { PP no } \\
\text { período(1) }^{(1)}\end{array}$} & \multirow{2}{*}{$\operatorname{VPM}(\mathrm{LBD})^{(1)}$} & \multirow{2}{*}{ VPM (LCD) ${ }^{(1)}$} & \multicolumn{2}{|c|}{ N-total } & \multicolumn{2}{|c|}{$\mathrm{N}-\mathrm{NH}_{4}{ }^{+}$} & \multicolumn{2}{|c|}{$\mathrm{N}-\mathrm{NO}_{3}{ }^{-}$} \\
\hline & & & & LBD & LCD & LBD & LCD & LBD & LCD \\
\hline dia & $\mathrm{mm}$ & -1 & - & & & $-\mathrm{mg} \mathrm{L}$ & & & \\
\hline 1 & 16,2 & 5,97 & 7,50 & $1.334,82$ & $1.006,96$ & 651,00 & 96,60 & 0,17 & 0,39 \\
\hline 5 & 48,8 & 11,80 & 10,60 & $1.487,67$ & $1.852,78$ & 903,00 & 128,80 & 1,05 & 0,17 \\
\hline 9 & 78,8 & 29,86 & 26,30 & $1.494,64$ & $1.922,12$ & 975,10 & 207,90 & 1,67 & 0,12 \\
\hline 13 & 0 & 7,45 & 7,15 & $1.331,28$ & $1.815,74$ & 964,60 & 154,70 & 0,80 & 0,25 \\
\hline 27 & 0,2 & 1,25 & 0,63 & $1.764,79$ & $1.902,87$ & $1.040,20$ & 364,70 & 1,29 & 1,05 \\
\hline 36 & 5,6 & 1,24 & 1,21 & 301,44 & 182,74 & 298,90 & 108,50 & 0,24 & 0,21 \\
\hline \multirow[t]{4}{*}{48} & 47,4 & 14,00 & 14,55 & 903,22 & 335,03 & 749,70 & 182,00 & 0,20 & 0,24 \\
\hline & \multicolumn{3}{|c|}{ Total Percolado (mg) } & $92.479,60$ & $105.691,02$ & $61.171,62$ & $12.460,04$ & 71,27 & 14,34 \\
\hline & \multicolumn{3}{|c|}{ Percolação média $\left(\mathrm{mg} \mathrm{dia}^{-1}\right)^{(2)}$} & $1.926,66$ & $2.201,90$ & $1.274,41$ & 259,58 & 1,48 & 0,30 \\
\hline & \multicolumn{3}{|c|}{ Percolação (g Mg $\left.{ }^{-1} \mathrm{MS} a^{-1}\right)^{(3)}$} & $9.864,75$ & $10.680,64$ & $6.525,14$ & $1.259,15$ & 7,60 & 1,45 \\
\hline
\end{tabular}

(1) TI - tempo de incubação; PP - precipitação pluvial; VMP - volume percolado médio; LBD - lodo bruto descoberto; LCD - lodo alcalinizado descoberto. ${ }^{(2)}$ Correlação entre PP no período e volume percolado $=0,93 .{ }^{\left({ }^{3}\right)}$ Estimativa para uma precipitação pluvial anual de 1.513 mm (média 1992-2002, SIMEPAR). O tratamento LCC não apresentou percolação devido à cobertura. 
(a)

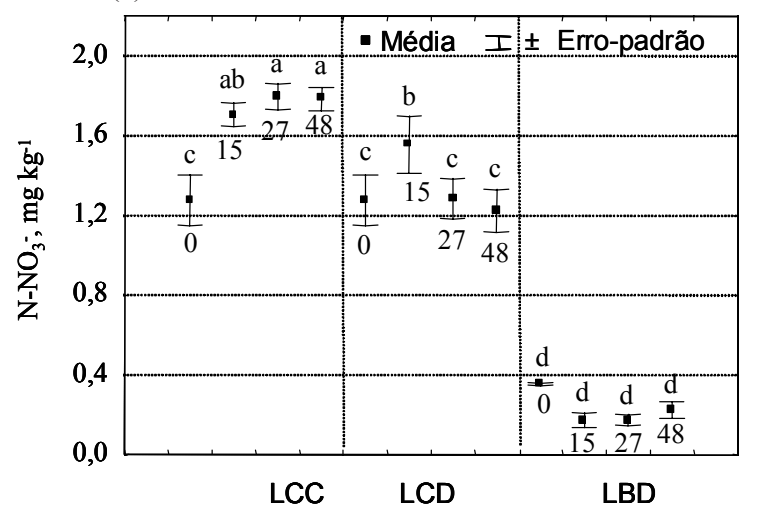

(b)

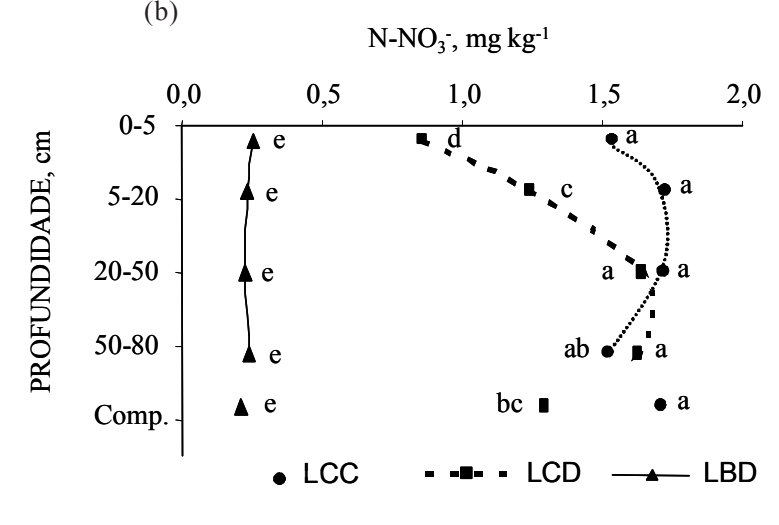

LCC - lodo alcalinizado coberto; LCD - lodo alcalinizado descoberto; LBD - lodo bruto descoberto. Comp: amostragem composta do perfil.

Médias seguidas pela mesma letra não diferem estatisticamente pelo teste de Duncan ${ }_{(0,05)}$.

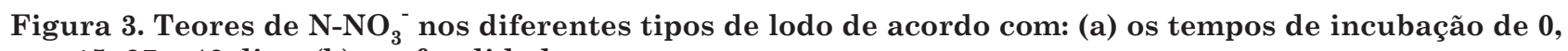
15, 27 e 48 dias; (b) profundidade.

na coluna, alternando teores em razão da profundidade, que necessitam ser mais bem estudados (Figura 3b). No tratamento LBD, os teores de $\mathrm{N}_{-} \mathrm{NO}_{3}$ - não variaram para os fatores tempo e profundidade, evidenciando a baixa atividade de nitrificação em $\mathrm{pH}$ ácido. Mesmo com leve acréscimo no $\mathrm{pH}$ no decorrer do tempo, o LBD não acusou efetivamente aumento nos teores de $\mathrm{N}-\mathrm{NO}_{3}{ }^{-}$. Adicionalmente, embora não-significativa, é possível verificar leve diminuição nos teores de $\mathrm{N}_{-} \mathrm{NO}_{3}{ }^{-}$entre os tempos 0 e 15 dias, período de maior precipitação. Em relação à profundidade, os menores teores nas camadas de 0-5 e 5-20 cm foram atribuídas à maior volatilização em superfície pela incidência da radiação solar direta e à percolação da água de chuva, causando maior lixiviação nos níveis superiores.

\section{Implicações relacionadas com a mineralização}

No presente estudo LBD apresentou as maiores taxas de mineralização, em torno de $4 \%$, comparativamente a $1,6 \%$ de LCD e $<1 \%$ para LCC, considerado apenas o período experimental. A taxa de mineralização de $\mathrm{N}$ recebe influência de muitas variáveis, especialmente climáticas, microbianas e de composição dos materiais (Russel, 1973; Keeney \& Nelson, 1982). Em razão dos níveis de umidade, aeração, porosidade (compactação por $\mathrm{CaO}$ ), tamanho de partículas, diversidade e especificidade microbiana e elevado teor de CO do lodo testado, ocorreram possivelmente oscilações na intensidade das atividades oxidativas dos microrganismos, o que pode ser verificado no tratamento LBD, com variações nos teores de $\mathrm{N}-\mathrm{NH}_{4}{ }^{+}$ (Figura 2a). O regime pluviométrico condicionou LBD e LCD a níveis de umidade elevados durante boa parte do período experimental.
Em LBD houve incrementos (ainda que com variações) na taxa de mineralização de acordo com o tempo, com predominância de formas reduzidas de $\mathrm{N}$, devidas ao desfavorecimento do meio à nitrificação, especialmente $\mathrm{pH}$ e $\mathrm{O}_{2}$ (Figuras 2a e 3a). Nos lodos alcalinos, LCC e LCD, a mineralização foi bastante reduzida [redução nos valores de $\mathrm{N}-\mathrm{NH}_{4}{ }^{+}$ e baixos teores de N-NO${ }_{3}^{-}$(Figuras 2a e b, 3a e b), respectivamente], especialmente após a alcalinização, pela expressiva elevação de $\mathrm{pH}$ e temperatura ocasionada pelo processo exotérmico.

Considerando que a partir de $40{ }^{\circ} \mathrm{C}$ a mineralização fica condicionada apenas à decomposição por organismos termófilos, tendo ainda o inconveniente do $\mathrm{pH}$ próximo a 12 (Figura 1a), ocorreu forte restrição à atividade microbiana. Ademais, é preciso ainda considerar a influência da composição físicoquímica da matriz quanto à biodegradabilidade e biodisponibilidade, à baixa evolução de $\mathrm{O}_{2}$ para situações de umidade na capacidade de campo, à temperatura ambiente relativamente baixa $\left(\chi=14,5^{\circ} \mathrm{C}\right)$, ao teor de umidade elevado e à especificidade da população microbiana.

O N também é consumido no processo de mineralização, sendo essencial ao crescimento e decomposição microbiana, com um ótimo para relação $\mathrm{C}: \mathrm{N}$ de 10:1. Os lodos domésticos, em geral, apresentam relações $\mathrm{C}: \mathrm{N}$ inferiores a 10:1, mesmo que alcalinizados, ocasionando maior imobilização do N. Quanto à possível influência de metais pesados presentes nos lodos, prejudicando a atividade microbiana, conforme relataram Chang \& Broadbent (1982) e Munn et al. (2000), historicamente, a estação de tratamento amostrada não tem apresentado indícios de concentrações elevadas destes metais tampouco ocorrem contribuições significativas de efluentes industriais. 


\section{Considerações sobre a porção lixiviada}

As formas amoniacais apresentaram-se de forma muito mais significativa nas águas de lixiviação, comparativamente aos teores de $\mathrm{N}_{-} \mathrm{NO}_{3}{ }^{-}$, correspondendo a aproximadamente $64 \%$ em LBD e $14 \%$ em LCD do N-total percolado (Quadro 2). Considerando estes valores e a reduzida percolação de $\mathrm{N}-\mathrm{NO}_{3}{ }^{-}$, boa parte do $\mathrm{N}$ no percolado está na forma orgânica, principalmente em LCD, onde a mineralização foi menor.

Em lodos brutos estocados, a lixiviação determina a principal fonte de perda de $\mathrm{N}$; em lodos alcalinizados, a volatilização de $\mathrm{NH}_{3}$ também assume grande importância. No presente estudo, estimativas em razão da pluviosidade média anual mostraram quantidades lixiviadas na ordem de 10,6 e 9,8 $\mathrm{kg} \mathrm{Mg}^{-1} \mathrm{MS}$ ano-1 de N-total, para LBD e LCD, respectivamente (Quadro 2). Os tratamentos LCD e LBD mostraram variações nos teores do extrato lixiviado em relação ao tempo, especialmente entre o $26^{\circ}$ e o $37^{\circ}$ dia, que podem estar relacionados com as oscilações na atividade microbiana e com a precipitação pluvial incidente, visto que a intensidade da precipitação deve exercer maior influência sobre a quantidade percolada do que o volume de precipitação, obviamente, considerando o extrato testado um meio de alta porosidade.

A capacidade de retenção de cátions por argilas e matéria orgânica em solos é de modo geral maior que a aniônica; deste modo, os nitratos não adsorvidos pelos colóides de carga negativa ficam mais sujeitos à lixiviação. No entanto, em virtude da alcalinização com $\mathrm{CaO}$, é possível que tenha ocorrido uma elevada saturação dos pontos de adsorção de $\mathrm{N}-\mathrm{NH}_{4}{ }^{+}$por $\mathrm{Ca}^{2+}$, determinando uma considerável lixiviação de $\mathrm{N}-\mathrm{NH}_{4}{ }^{+}$. Ademais, o elevado pH leva ao aumento na taxa de hidrólise e desestabilização de estruturas amoniacais, como, por exemplo $\left(\mathrm{NH}_{4}\right)_{2} \mathrm{CO}_{3}$. Isto pode ser inferido pela lixiviação de $\mathrm{N}-\mathrm{NH}_{4}{ }^{+}$no extrato que sofreu alcalinização. Porém, o extrato líquido LBD (sem alcalinização) apresentou teores ainda maiores de $\mathrm{N}-\mathrm{NH}_{4}{ }^{+}$, comparativamente a LCD (Quadro 2). Estudos posteriores são necessários para verificar os fatores envolvidos na lixiviação de $\mathrm{N}_{-} \mathrm{NH}_{4}{ }^{+}$em LBD, visto que não há adição de sais ou outros materiais alcalinos e ser o $\mathrm{pH}$ suficientemente baixo a ponto de não promover, em condições normais, grandes lixiviações do cátion. Um dos aspectos que notadamente pode explicar isto é a indicação de concentrações de $\mathrm{N}-\mathrm{NH}_{4}{ }^{+}$no extrato sólido, seis vezes maior em LBD que em LCD.

Assim, independentemente se alcalinizados ou brutos, os lodos estocados apresentam perdas consideráveis de formas minerais de $\mathrm{N}-\mathrm{NH}_{4}{ }^{+}$. Isto demonstra a importância da lixiviação deste íon, porém, certamente, em se tratando da adição de lodos aos solos, as implicações não têm a mesma pertinência. Prado Filho \& Selbach (1988), testando diferentes tipos de fertilização química e orgânica, constataram que as águas de escorrimento superficial não promoveram arrastes significativos das formas inorgânicas de $\mathrm{N}$, tendo $\mathrm{N}-\mathrm{NO}_{3}$ apresentado menor índice de escorrimento que a forma $\mathrm{N}-\mathrm{NH}_{4}{ }^{+}$.

Obviamente, os baixos valores de $\mathrm{N}_{-} \mathrm{NO}_{3}{ }^{-}$observados no lixiviado estão diretamente relacionados com os teores reduzidos na coluna e extrema adversidade à nitrificação. Embora as concentrações sejam reduzidas nos extratos, sólido e líquido (Figura 3a), é possível constatar a maior lixiviação em relação ao tempo no tratamento LCD em comparação a LCC, evidenciando o efeito da chuva. Em LBD, os valores são muito baixos e estatisticamente iguais.

Contudo, apesar dos baixos teores de $\mathrm{N}-\mathrm{NO}_{3}$ observados neste estudo, é preciso atentar quando da disposição destes lodos alcalinizados em solos, decorrente do alto teor de N-orgânico no material com potencial de nitrificação e a contaminação de lençóis. He et al., 2000 mostraram que os lodos domésticos têm um alto potencial de mineralização. Entretanto, quando alcalinizados, ocorre uma significativa redução na taxa de mineralização e, quando adicionados ao solo com a finalidade de condicionante agrícola, a atividade microbiana intensifica-se com o restabelecimento do $\mathrm{pH}$ em níveis satisfatórios para seu desenvolvimento.

\section{CONCLUSÕES}

1. As condições do meio ocasionadas pela alcalinização, pela adição de $\mathrm{CaO}$ na proporção de $50 \%$ MS lodo (pH e temperatura) e a umidade elevada reduziram significativamente a intensidade de mineralização de $\mathrm{N}$ e a nitrificação. A alcalinização reduziu os teores de N-total em até 50 \%, em decorrência do efeito diluição, volatilização de $\mathrm{NH}_{3}$ e lixiviação organomineral.

2. O tratamento LCC - com cobertura apresentou maiores teores de $\mathrm{NH}_{4}{ }^{+}$em superfície.

3. O processo de caleação potencializou perdas de $\mathrm{N}$ pela lixiviação de íons $\mathrm{N}-\mathrm{NH}_{4}{ }^{+}$. Igualmente, o tratamento sem alcalinização também apresentou significativas perdas de $\mathrm{N}-\mathrm{NH}_{4}{ }^{+}$por lixiviação que precisam ser mais bem investigadas.

\section{LITERATURA CITADA}

ADAMS, F. \& MARTIN, J.B. Liming effects on nitrogen use and efficiency. In: HAUCK, R.D., ed. Nitrogen in crop production. Madison, American Society of Agronomy, 1984. 804 p. 
ANDREOLI, C.V. \& CARNEIRO, C. Avaliação do comportamento vertical de $\mathrm{N}$ orgânico, $\mathrm{N}$ amoniacal e $\mathrm{pH}$ em leiras de lodo de esgoto caleado. R. Sanare, 18:63-70, 2002.

ANDREOLI, C.V.; LARA, A.I. \& FERNANDES, F. Reciclagem de biossólidos: transformando problemas em soluções. 2.ed. Curitiba, 2001. 300p.

BRADY, N.C. Natureza e propriedade dos solos. 7.ed. Rio de Janeiro, Freitas Bastos, 1989. 878p.

CHAE, Y.M. \& TABTABAI, M.A. Mineralization of nitrogen in soils amended with organic wastes. J. Environ. Qual., 15:193-198, 1986.

CHANG, F.H. \& BROADBENT, F.E. Influence of trace metals on some soil nitrogen transformations. J. Environ. Quallity, 11:1-4, 1982.

GOMES, F.P. Curso de estatística experimental. 12.ed., Piracicaba, 1987. 467p.

HAUCK, R.D. Significance of nitrogen fertilizer microsite reactions in soil. In: HAUCK, R.D., ed. Nitrogen in crop production. Madison, American Society of Agronomy, 1984. 804p.

HE, Z.L.; ALVA, A.K.; YAN, P.; LI; Y.C.; CALVERT, D.V.; STOFFELLA, P.J. \& BANKS, D.J. Nitrogen mineralization and transformation from composts and biosolids during field incubation in a sandy soil. Soil Sci., 65, 2000.

HEINZMANN, F.X.; MIYAZAVA, M. \& PAVAN, M.A. Determinação de $\mathrm{N}-\mathrm{NO}_{3}$ por espectrofotometria de absorção ultravioleta. R. Bras. Ci. Solo, 8:159-163, 1984.

ILHENFELD, R.G.K.; PEGORINI, E.S. \& ANDREOLI, C.V. Fatores limitantes. In: PROSAB - Programa de Pesquisa em saneamento Básico. Rio de Janeiro, 1999a. 97p.

ILHENFELD, R.G.K.; ANDREOLI, C.V. \& LARA, A.I. Higienização do lodo de esgoto. In: PROSAB - Programa de Pesquisa em saneamento Básico. Rio de Janeiro, 1999b. 97p.

KEENEY, D.R. \& NELSON, D.W. Nitrogen-inorganic forms. In: PAGE, A.L. ??DEMAIS EDITORES, eds. Agronomy Monogr., 9, ASA and SSSA, Madison, 1982. Part 2, p.643698.

MELO, W.J. \& MARQUES, M.O. Potencial do lodo de esgoto como fonte de nutrientes para as plantas. In: BETTIOL, W. \& CAMARGO, O.A., eds. Impacto ambiental do uso agrícola do lodo de esgoto. Jaguariúna, São Paulo, Embrapa Meio Ambiente, 2000. 312p.
MUNN, K.J.; EVENS, J. \& CHALK, P.M. Mineralization of soil and legume nitrogen in soils treated with metalcontaminated sewage sludge. Soil Biol. Biochem., 32:2031$2043,2000$.

NORMAN, R.J. \& STUCKI, J.W. The determination of nitrate and nitrite in soil extracts by ultraviolet spectrophotometry. J. Soil Sci. Soc. Am., 45:347-353, 1981.

PRATT, P.F. Nitrogen use e nitrate leaching in irrigated agriculture. In: HAUCK, R.D., ed. Nitrogen in crop production. Madison, American Society of Agronomy, 1984. 804p.

PRADO FILHO, J.F. \& SELBACH, P.A. N inorgânico em águas de escorrimento superficial. R. CETESB Tecn. Amb., 2:8487, 1988.

RUSSEL, E.W. Soil conditions and plant growth. 10.ed. London, 1973. 849p.

SOMMERS, L.E. \& GIODARNO, P.M. Use of nitrogen from agricultural, industrial, and municipal wastes. In: HAUCK, R.D., ed. Nitrogen in crop production. Madison, American Society of Agronomy, 1984. 804p.

TCHOBANOGLOUS, G.; BURTON, F.L. \& STENSEL, H.D. Wastewater engineering: Treatment, disposal and reuse. 4.ed. New York, McGraw Hill, 2003. 1848p.

TEDESCO, M.J.; GIANELLO C.; BISSANI, C.A.; BOHNEN, H. \& VOLKWEISS, S.J. Análises de solo, plantas e outros materiais. 2.ed. Universidade Federal do Rio Grande do Sul, 1995. 174p.

TRIOLA, M.F. Introdução à estatística. Rio de Janeiro, LTC, 1999. 410p.

WILLIAMS, J.R. \& KISSEL, D.E. Water percolation: an indicator of nitrogen-leaching potencial. In: FOLLET, R.F.; KEENEY, D.R. \& CRUSE, R.M., eds. Managing nitrogen for groundwater quality and farm profitability. Madinson, Soil Science Society of America, 1991. 357p.

ZEBARTH, B.J.; McDOUGALL, R.; NEILSEN, G. \& NEILSEN, D. Availability of nitrogen from municipal biosolids for dryland forage grass. Can. J. Plant Sci., 80:575-582, 2000. 